\title{
RESEARCH
}

Open Access

\section{Metformin attenuates plaque-associated tau pathology and reduces amyloid- $\beta$ burden in APP/PS1 mice}

Yanxing Chen ${ }^{1 \dagger}$, Shuai Zhao ${ }^{1 \dagger}$, Ziqi Fan ${ }^{1}$, Zheyu Li ${ }^{1}$, Yueli Zhu ${ }^{1,2}$, Ting Shen ${ }^{1}$, Kaicheng Li ${ }^{1}$, Yaping Yan ${ }^{1}$, Jun Tian ${ }^{1}$, Zhirong Liu' ${ }^{1}$ and Baorong Zhang ${ }^{1 *}$ (D)

\begin{abstract}
Background: The neuropathological hallmarks of Alzheimer's disease (AD) are amyloid- $\beta(A \beta)$ plaques and neurofibrillary tangles (NFTs). The amyloid cascade theory is the leading hypothesis of AD pathology. A $\beta$ deposition precedes the aggregation of tau pathology and $A \beta$ pathology precipitates tau pathology. Evidence also indicates the reciprocal interactions between amyloid and tau pathology. However, the detailed relationship between amyloid and tau pathology in AD remains elusive. Metformin might have a positive effect on cognitive impairments. However, whether metformin can reduce AD-related pathologies is still unconclusive.

Methods: Brain extracts containing tau aggregates were unilaterally injected into the hippocampus and the overlying cerebral cortex of 9-month-old APPswe/PS1DE9 (APP/PS1) mice and age-matched wild-type (WT) mice. Metformin was administrated in the drinking water for 2 months. A $\beta$ pathology, tau pathology, plaque-associated microgliosis, and autophagy marker were analyzed by immunohistochemical staining and immunofluorescence analysis 2 months after injection of proteopathic tau seeds. The effects of metformin on both pathologies were explored.
\end{abstract}

Results: We observed tau aggregates in dystrophic neurites surrounding $A \beta$ plaques (NP tau) in the bilateral hippocampi and cortices of tau-injected APP/PS1 mice but not WT mice. A $\beta$ plaques promoted the aggregation of NP tau pathology. Injection of proteopathic tau seeds exacerbated A $\beta$ deposits and decreased the number of microglia around A $\beta$ plaques in the hippocampus and cortex of APP/PS1 mice. Metformin ameliorated the microglial autophagy impairment, increased the number of microglia around $A \beta$ plaques, promoted the phagocytosis of NP tau, and reduced A 3 load and NP tau pathology in APP/PS1 mice.

Conclusion: These findings indicate the existence of the crosstalk between amyloid and NP tau pathology. Metformin promoted the phagocytosis of pathological $A \beta$ and tau proteins by enhancing microglial autophagy capability. It reduced A $\beta$ deposits and limited the spreading of NP tau pathology in APP/PS1 mice, which exerts a beneficial effect on both pathologies.

Keywords: A $\beta$ pathology, Tau pathology, Spread, Metformin, Microglia, Alzheimer' disease

\footnotetext{
*Correspondence: brzhang@zju.edu.cn

${ }^{\dagger}$ Yanxing Chen and Shuai Zhao contributed equally to this work.

'Department of Neurology, The Second Affiliated Hospital, School of Medicine, Zhejiang University, Hangzhou, People's Republic of China

Full list of author information is available at the end of the article
} 


\section{Introduction}

Alzheimer's disease (AD) is the most common cause of dementia, posing a heavy burden for society. It is characterized by the deposition of extracellular amyloid plaques and the formation of intracellular neurofibrillary tangles (NFTs). The amyloid cascade theory, which is considered as the dominant hypothesis of $\mathrm{AD}$, states that $A \beta$ accumulation is the initial event in the pathogenesis of $\mathrm{AD}$, resulting in the formation of NFTs and neuronal death of AD [1]. However, accumulating studies suggest a mutual interaction between $A \beta$ and tau pathology. Studies in animal models showed that $A \beta$ plaques could enhance tau seeding and pathology [2-4]. It was found that injection of human AD-brain-derived proteopathic tau (AD-tau) into the brains of plaque bearing transgenic mice facilitated the spreading of tau pathology, which initially appeared as NP tau, and then followed by the formation of NFTs and neuropil threads (NTs) [2]. Another study also observed augmented spreading of tau pathology in 5xFAD mice with injection of AD-tau [3]. App/MAPT double-knock-in mice exhibited higher tau phosphorylation than did single $M A P T$ knock-in mice, indicating the enhancement of $A \beta$ on tau pathology [4].

It is known that tau pathology correlates more strongly with cognitive decline in $\mathrm{AD}$ patients than amyloid pathology $[5,6]$. NFTs appear in a hierarchical and stereotypical fashion, beginning in the transentorhinal cortex and later spreading to brain regions such as the hippocampus and, eventually, the cortex [7]. Mounting evidence suggests that tau aggregates recruit monomeric tau into fibrillar aggregates that spread to other brain regions which is called "tau propagation" $[8,9]$.

In recent years, a link between diabetes and AD has been largely accepted. Type 2 diabetes mellitus (T2DM) is associated with cognitive impairment and an increased risk of $\mathrm{AD}$ [10]. Impaired glucose metabolism in the brain is detected early in $\mathrm{AD}$ [11], and $\mathrm{AD}$ patients have insulin resistance in the brain [12]. Metformin is widely used for the treatment of T2DM. Growing evidence suggests that it might benefit age-related diseases such as cancer, cardiovascular disease, and neurodegenerative diseases, such as AD [13]. However, the effects of metformin on cognition are still controversial. Epidemiological studies have shown that metformin usage is associated with significantly lower risk of cognitive dysfunction in older adults with T2DM in both crosssectional analysis and in longitudinal analysis [14-16]. An interventional study found that, in overweight patients aged 55 to 90 years with amnestic mild cognitive impairment, those receiving metformin for 12 months had better performance in the selective reminding test than those receiving placebo [17]. However, a casecontrol study reported that long-term treatment of metformin ( $\geq 60$ prescriptions, an average prescription covers 45-90 days of treatment) was associated with higher risk of $\mathrm{AD}$ in $\mathrm{T} 2 \mathrm{DM}$ patients over age 65, although there was no consistent trend with increasing number of prescriptions [18]. It was suggested that the effects of metformin on cognition may differ relying on the risk profile of the patients [19]. Mechanistic studies have also been carried out to unveil the effect on metformin on AD pathologies. Protective effect of metformin on cognitive impairment in different models of diabetes has been reported [20-22]. Of note, it is reported that metformin could reduce tau phosphorylation in different mouse models including $\mathrm{AD}[21,23,24]$. However, increased insoluble tau species was also reported in another study [25]. Further studies are still in need to elucidate whether metformin could exert beneficial effects on AD pathologies.

In this study, we seeded APP/PS1 mice with tau seeds to investigate the effects of amyloid pathology on tau seeding activity. The effects of metformin on amyloid pathology and tau spreading were also investigated. We observed that $\mathrm{A} \beta$ plaque promoted NP tau aggregation. Injection of proteopathic tau seeds exacerbated $A \beta$ deposits and decreased the number of microglia around $\mathrm{A} \beta$ plaques. Administration of metformin ameliorated the microglial autophagy impairment, enhanced the activation of microglia around $A \beta$ plaques, promoted the phagocytosis of NP tau, and attenuated A $\beta$ load and NP tau aggregation.

\section{Methods}

\section{Animals and drug treatment}

All procedures were approved by the Institutional Animal Care and Use Committee of Zhejiang University and were performed in accordance with the National Institutes of Health Guide for the Care and Use of Laboratory Animals guidelines for the ethical treatment of animals. Efforts were made to minimize the number of animals used. APP/PS1 transgenic mice were purchased from Model Animal Research Center of Nanjing University (Nanjing, China). Mice were housed in filtered cages in a temperature-controlled room with a 12:12 light/dark cycle with free access to food and water.

At 9 months of age, female APP/PS1 mice were randomly assigned to four groups: (1) APP/PS1 mice injected with brain extract from WT control mice ( ${ }^{\mathrm{WT}} \mathrm{BE}$ ) and treated with vehicle (ctr+veh group, $n=5$ ); (2) APP/PS1 mice injected with brain extract containing tau aggregates from PS19 mice $\left({ }^{\mathrm{PS} 19} \mathrm{BE}\right)$ and treated with vehicle (tau+veh group, $n=5$ ); (3) APP/PS1 mice injected with ${ }^{\mathrm{WT}} \mathrm{BE}$ and treated with metformin (ctr+ met group, $n=5)$; (4) APP/PS1 mice injected with ${ }^{\mathrm{PS} 19} \mathrm{BE}$ and treated with metformin (tau+met group, $n=5$ ). Another group of WT mice injected with ${ }^{\mathrm{PS} 19} \mathrm{BE}$ and treated with vehicle (tau+veh group, $n=5$ ) were also 
included. Mice in the treatment group received $4 \mathrm{mg} / \mathrm{ml}$ metformin (Sigma, St. Louis, MO, USA) in the drinking water for 2 months. Mice in the vehicle group received normal drinking water. Drinking bottles were replenished with fresh water or metformin solution every week.

\section{Preparation of brain extracts}

PS19 mice were obtained from the Jackson Laboratory (B6;C3-Tg (Prnp-MAPT*P301S)PS19Vle/J; stock number, 008169, New Harbor, ME, USA). Brain extracts were prepared from end-stage PS19 mice and agematched WT mice as previously described [26]. Briefly, mice were sacrificed by dislocation of the neck and decapitation. Cerebral cortices and hippocampi were snap frozen in dry ice. Tissues were combined and homogenized at $10 \%(\mathrm{w} / \mathrm{v})$ in sterile phosphate-buffered saline (PBS), followed by sonication and centrifugation at $3000 \mathrm{~g}$ at $4{ }^{\circ} \mathrm{C}$ for $5 \mathrm{~min}$. The supernatant was stored at $-80^{\circ} \mathrm{C}$ until use.

\section{Steoretaxic injection}

Mice were deeply anesthetized with $1.25 \%$ Avertin (Sigma, St. Louis, MO, USA). Intracerebral injections were performed as described previously [26] and is shown in Fig. 1. In brief, mice were placed in a stereotaxic frame and a heating blanket with a rectal probe to maintain body temperature at $38 \pm 1{ }^{\circ} \mathrm{C}$. Once the skull was exposed and cleaned, a hole was drilled over the appropriate coordinates and brain extracts were unilaterally infused using a Hamilton syringe. The hippocampus (bregma, $-2.50 \mathrm{~mm}$; lateral, $+2.00 \mathrm{~mm}$; and depth, $-1.80 \mathrm{~mm}$ ) and then the overlying cerebral cortex (bregma, $-2.50 \mathrm{~mm}$; lateral, $+2.00 \mathrm{~mm}$; and depth, $-0.80 \mathrm{~mm}$ ) were injected with $2.5 \mu \mathrm{l}$ of inoculum at a flow rate of $1.25 \mu \mathrm{l} / \mathrm{min}$ and the needle was kept in position for additional $3 \mathrm{~min}$ before slow withdrawal. Mice were allowed to completely recover on a heating pad before they were returned to their home cages.

\section{Immunohistochemistry and immunofluorescence}

Two months after treatment, mice were deeply anesthetized and transcardially perfused with PBS followed by buffered $4 \%$ paraformaldehyde. The whole brains were collected, post-fixed in buffered $4 \%$ paraformaldehyde overnight at $4{ }^{\circ} \mathrm{C}$, and dehydrated in buffered $30 \%$ sucrose solution. The brains were then cut into $30-\mu \mathrm{m}$ serial coronal sections on a cryostat and the free-floating sections were preserved in anti-freeze solution (glycerol, ethylene glycol in $0.1 \mathrm{M} \mathrm{PBS}$ ) at $-20^{\circ} \mathrm{C}$ until use.

Immunohistochemistry was performed according to our previously described methods [27]. Briefly, sections were incubated with $0.3 \% \mathrm{H}_{2} \mathrm{O}_{2}$ for $20 \mathrm{~min}$ and $0.3 \%$ Triton X-100 for $15 \mathrm{~min}$ at room temperature, washed in PBS, and blocked in 5\% normal goat serum with $0.1 \%$ Triton X-100 for $30 \mathrm{~min}$. Sections were then incubated overnight with anti-A $\beta$ antibody (6E10) (1:500 dilution; Biolegend, San Diego, CA, USA) at $4{ }^{\circ} \mathrm{C}$. Sections were washed and incubated with horseradish peroxidaseconjugated secondary antibody and visualized with a stable diaminobenzidine/hydrogen peroxide solution. Stained sections were then mounted on microscope slides, dehydrated through graded alcohols, cleared in xylene, and sealed with neutral balsam.

For immunofluorescence, sections were permeabilized with $0.3 \%$ Triton X-100, blocked with 5\% normal goat serum for $30 \mathrm{~min}$, and then incubated with the following primary antibodies overnight at $4{ }^{\circ} \mathrm{C}$ : anti-A $\beta$ antibody (6E10) (1:500 dilution; Biolegend, San Diego, CA, USA), anti-A $\beta$ antibody (4G8) (1:500 dilution; Biolegend, San Diego, CA, USA), anti-AT8 antibody (phospho-tau at Ser202/Thr 205; 1:500 dilution; Thermo Fisher Scientific, MA, USA), anti-AT180 antibody (phospho-tau at Thr231; 1:500 dilution; Thermo Fisher Scientific, MA, USA), anti-phospho-Tau (Ser422) antibody (1:500 dilution; Thermo Fisher Scientific, MA, USA), anti-Iba1 antibody (1:500 dilution; Wako Chemicals, Richmond, VA, USA), and anti-p62 antibody (1:250 dilution; Cell Signaling Technology, Danvers, MA). Normal goat serum in the absence of primary antibody was used as a
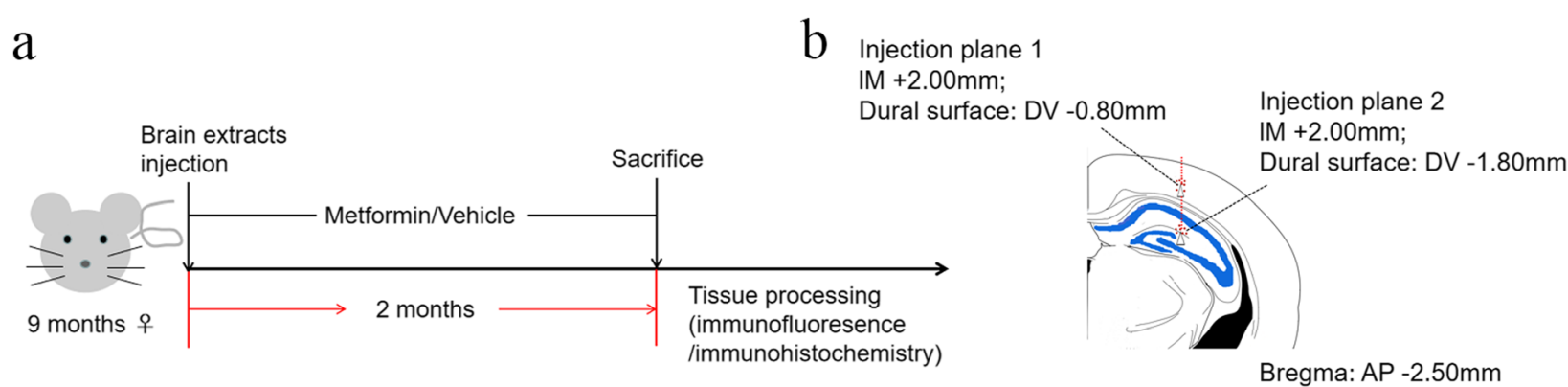

Fig. 1 Schematic illustrations of injection sites with coronal planes. a Study design. b Red dotted line indicates injection path and white arrowheads indicate injection site 
negative control. The next morning, sections were incubated with an appropriate secondary antibody for $1 \mathrm{~h}$ in darkness. Then, the sections were incubated with 4',6diamidino-2-phenylindole (DAPI) for $5 \mathrm{~min}$, washed in PBS, and mounted on microscope slides with SlowFade Gold antifade reagent.

Numbers of AT8+ NP tau was analyzed as previously described [2]. In brief, NP tau was defined as a cluster of granule which was AT8+ without a single central nucleus. Four coronal sections of the same reference position from each mouse were selected and measured through blinded manual counting. Quantification of $A \beta$ plaque was performed according to our previously described methods [28]. Briefly, four sections were selected from each mouse using the ImageJ software $(\mathrm{NIH}, \mathrm{Be}-$ thesda, MD, USA). The $A \beta$ plaque burden (\% of area) was calculated relative to the total area of the selected region $($ area $\%=$ plaque area/total area selected $\times 100 \%)$. Number of plaque-associated microglia was analyzed as previously described [29]. Briefly, to assign spots to each microglial cell body, the same threshold was applied in all images. 6E10 surfaces were dilated $15 \mu \mathrm{m}$, and spots of microglia were counted manually in the expanded area. Any spots completely within or partially in the extended area were counted. The percentage of the number of Iba1+ microglia containing AT8+ aggregates was calculated relative to total number of Iba1+ cells selected. Quantification of p62 was performed as previously described [30]. Briefly, the p62+ area (\% of area) was calculated relative to the total area of the selected region $($ area $\%=p 62+$ area/total area selected $\times 100 \%)$. The percentage of $\mathrm{p} 62+/ \mathrm{Iba} 1+$ area was calculated relative to the total Iba1+ area selected, and the percentage of the number of Iba1+ microglia containing p62+ aggregates was calculated relative to total number of Iba1+ cells selected. All counts were performed in a blinded fashion.

\section{Statistical analysis}

The GraphPad Prism software (GraphPad Software Inc., San Diego, CA, USA) was used for statistical analysis. For comparisons among various groups, results were analyzed by one-way analyses of variance (ANOVA) followed by the Bonferroni post hoc test. For comparisons between two groups, results were analyzed by the Student's $t$ test. Data are shown as mean \pm standard error of the mean (SEM), and $P$ values $<0.05$ were considered statistically significant.

\section{Results}

Injection of proteopathic tau seeds induced NP tau aggregation in APP/PS1 mice

Two months following the injection of ${ }^{\mathrm{PS} 19} \mathrm{BE}$ into APP/ PS1 mice (Fig. 1), we observed clusters of AT8+ tau particles surrounding $6 \mathrm{E} 10+\mathrm{A} \beta$ plaques (Fig. 2a, b), resembling NP tau in AD patients. Similar results were observed with antibody $4 \mathrm{G} 8$ for $\mathrm{A} \beta$ plaques and antibodies AT180 and p-Tau 422 for NP tau staining (Fig. 3). NP tau distributed mainly in the dentate gyrus (DG) and $\mathrm{CA} 3$ region of the ipsilateral hippocampus of ${ }^{\mathrm{PS} 19} \mathrm{BE}$ injected APP/PS1 mice. NP tau was observed not only in the ipsilateral hippocampus and cortex, but also the contralateral hippocampus and cortex in ${ }^{\mathrm{PS} 19} \mathrm{BE}$-injected APP/PS1 mice, which suggests propagation of tau pathology to the contralateral brain. On the contrary, NP tau pathology was absent in ${ }^{\mathrm{WT}} \mathrm{BE}$-injected APP/PS1 mice (Fig. 2c) or ${ }^{\mathrm{PS} 19} \mathrm{BE}$-injected WT mice (Fig. 2d), indicating that proteopathic tau seeds and $\mathrm{A} \beta$ burden are both necessary for the aggregation of NP tau. We did not find NFTs in any group of mice.

\section{Injection of proteopathic tau seeds exacerbated $A \beta$ burden in APP/PS1 mice}

To better understand the relationship between $A \beta$ pathology and tau pathology, we examined the $A \beta$ plaque burden in APP/PS1 mice. We found much more A $\beta$ burden in the bilateral cortices (Fig. 4a) and hippocampi (Fig. 5a) of ${ }^{\text {PS19 }}$ BE-injected APP/PS1 mice than that of ${ }^{W T}$ BE-injected APP/PS1 mice. Moreover, increased A $\beta$ plaque burden was especially obvious in the dentate gyrus and CA3 subfields of the ipsilateral hippocampus of ${ }^{\mathrm{PS} 19}$ BE-injected APP/PS1 mice (Fig. 5d-h).

\section{Injection of proteopathic tau seeds reduced microgliosis around $A \beta$ plaques in APP/PS1 mice}

To explore the underlying mechanisms of increased $A \beta$ accumulation, we investigated the involvement of microglia in APP/PS1 mice. We observed significantly decreased numbers of microglia surrounding $A \beta$ plaques in the ${ }^{\mathrm{PS} 19} \mathrm{BE}$-injected APP/PS1 mice as compared to the ${ }^{\mathrm{WT}} \mathrm{BE}$-injected APP/PS1 mice (Fig. 6a, b). The results showed that injection of proteopathic tau seeds decreased plaque-associated microgliosis.

\section{Metformin reduced $A \beta$ burden in APP/PS1 mice}

To examine the effect of metformin on $A \beta$ plaques in the brain, we analyzed $A \beta$ plaque deposits in the brains of APP/PS1 mice. In the ${ }^{\mathrm{PS} 19} \mathrm{BE}$-injected APP/PS1 mice, metformin significantly reduced $A \beta$ plaques in bilateral cortices and the contralateral hippocampus of the mice (Figs 4 and 5). A $\beta$ plaque burden was further analyzed in the subfields of the hippocampus. We found decreased $\mathrm{A} \beta$ burden in bilateral dentate gyrus and CA3 subfields of the hippocampus of metformin-treated mice than that of the vehicle-treated mice. No significant differences of $\mathrm{A} \beta$ burden in the CA1 subfield of the hippocampus between the groups were observed. In the ${ }^{\mathrm{WT}} \mathrm{BE}$-injected APP/PS1 mice, metformin decreased $A \beta$ burden in the 

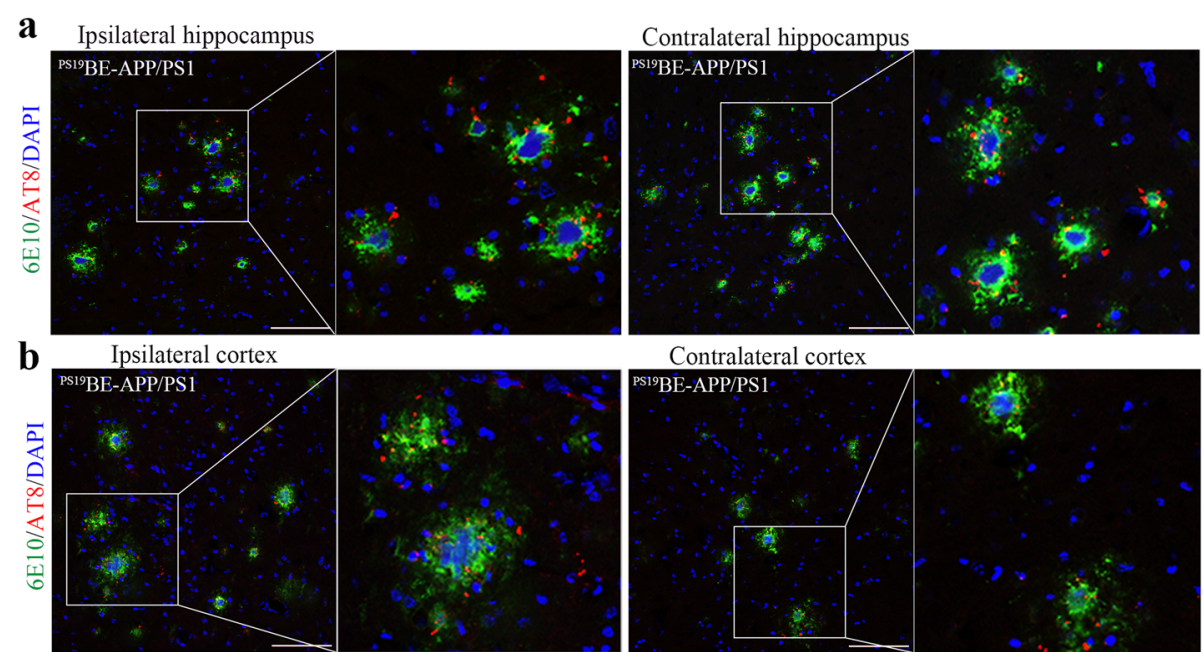

Contralateral cortex

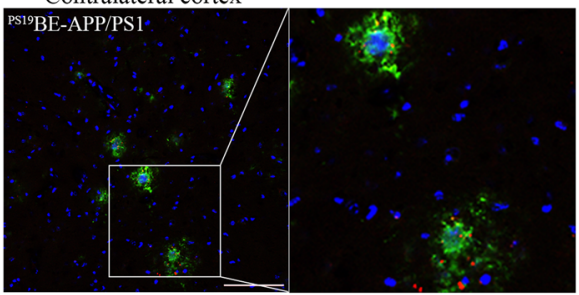

C Ipsilateral hippocampus

Ipsilateral cortex
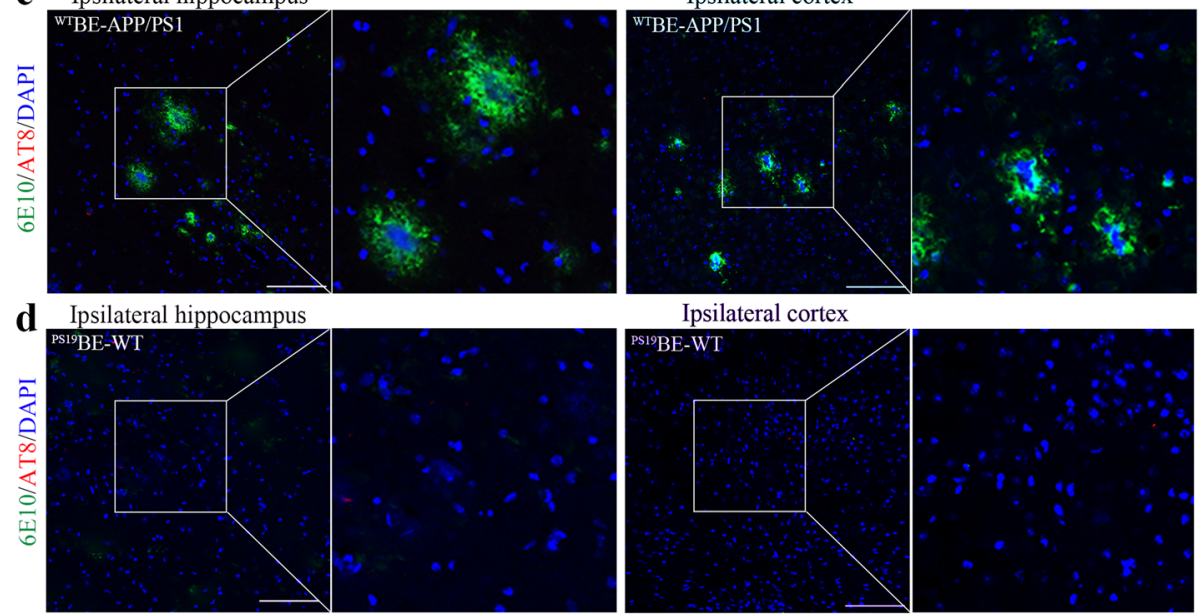

Ipsilateral cortex

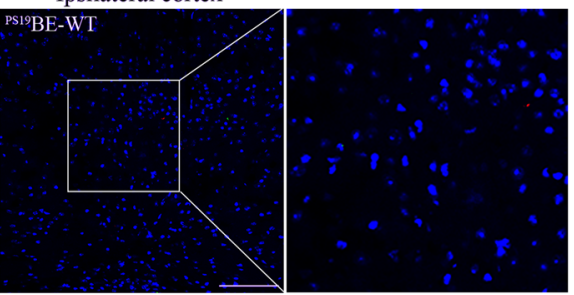

Fig. 2 Injection of proteopathic tau seeds induced NP tau aggregation in APP/PS1 mice. a-d Representative images of co-staining for 6E10 (green), AT8 (red) in the hippocampus, and cortex of ${ }^{\text {PS19 }}{ }^{B E}$-injected APP/PS1 mice (a, b), ${ }^{\text {WT } B E-i n j e c t e d ~ A P P / P S 1 ~ m i c e ~(c), ~ a n d ~}{ }^{\text {PS19} B E-i n j e c t e d ~ W T ~}$ mice (d). Partial magnifications are shown. Scale bar, $100 \mu \mathrm{m}$

bilateral dentate gyrus and ipsilateral CA3 subfields of hippocampus (Fig. 5). These results demonstrated that metformin treatment ameliorated $A \beta$ plaque burden as well as pathologic tau-induced exacerbation of $A \beta$ accumulation in APP/PS1 mice.

\section{Metformin limited the spreading of NP tau aggregation in} ${ }^{\mathrm{PS} 19} \mathrm{BE}$-injected APP/PS1 mice

To explore the effect of metformin on the spreading of $\mathrm{NP}$ tau aggregation in ${ }^{\mathrm{PS} 19} \mathrm{BE}$-injected APP/PS1 mice, the amounts of NP tau labeled with antibodies AT8, AT180, or $\mathrm{p}$-Tau 422 surrounding individual $A \beta$ plaques labeled with antibodies 6E10 and 4G8 were quantified. The numbers of NP tau in metformin-treated mice were reduced in comparison to the vehicle-treated mice (Fig. 3), which implied that metformin limited the spreading of tau pathology in APP/PS1 mice.

\section{Metformin enhanced plaque-associated microgliosis in} APP/PS1 mice

To explore the possible mechanisms underlying the effects of metformin on $A \beta$ pathology, we investigated the involvement of microglia. Administration of metformin enhanced the plaque-associated microgliosis (Fig. 6a, b). Moreover, we found more activated microglial cells with larger, rounder somata and short, thick extensions surrounding $A \beta$ plaques in the brains of the metformintreated mice. Altogether, the data suggests that metformin effectively promoted the activation of microglia in ${ }^{\mathrm{PS} 19} \mathrm{BE}$-injected APP/PS1 mice.

\section{Metformin promoted the phagocytosis of NP tau} aggregates by microglia in ${ }^{\mathrm{PS} 19} \mathrm{BE}-$-injected APP/PS1 mice To explore the mechanisms underlying the decreased NP tau pathology in metformin-treated mice, colocalization between Iba1+ microglia and AT8+ seeds 


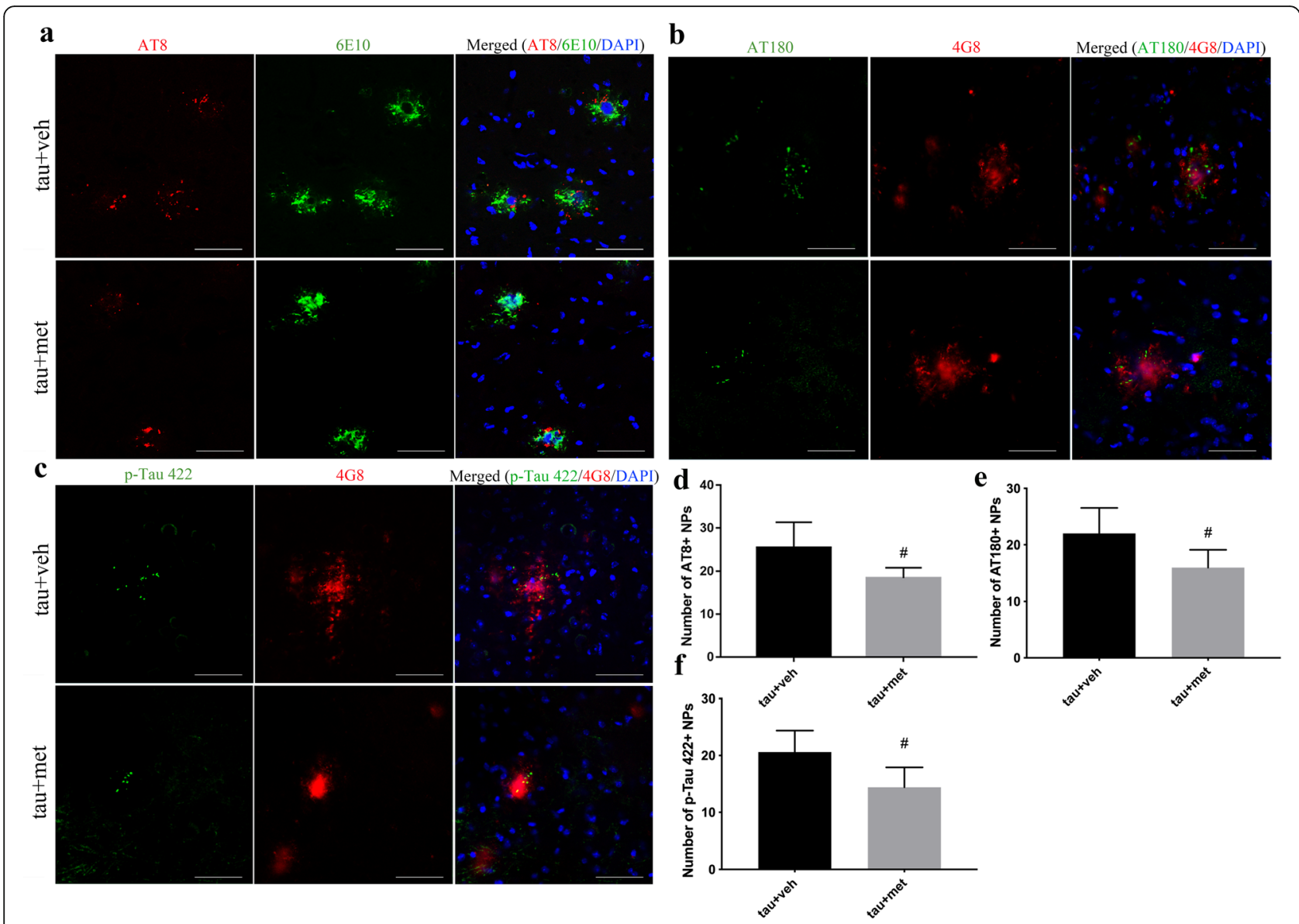

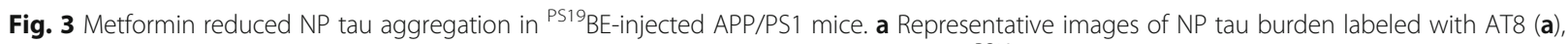

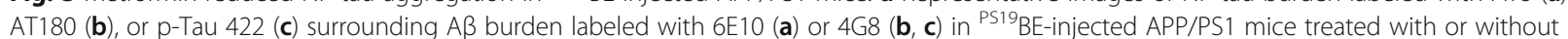
metformin. Scale bars, $50 \mu \mathrm{m}$. $\mathbf{d}$-f Quantification of NP tau in ${ }^{\mathrm{PS} 19} \mathrm{BE}$-injected APP/PS1 mice treated with or without metformin. Data were analyzed by Student's $t$ test. Values are presented as mean \pm SEM. $n=5$ per group. ${ }^{\#} P<0.05$ vs. tau+veh group

were investigated. We found internalized NP tau in microglia (Fig. 6c, d), indicating that microglia took up tau seeds in ${ }^{\mathrm{PS} 19} \mathrm{BE}$-injected APP/PS1 mice. This observation implies that metformin may promote the phagocytosis of tau seeds by microglia, thus reducing the NP tau pathology.

\section{Metformin reduced accumulation of autophagy-related protein in APP/PS1 mice}

Autophagy dysregulation has been reported in AD brain, which contributes to the formation of AD pathologies [31]. Therefore, we investigated the involvement of autophagy in the accumulation of $\mathrm{A} \beta$ plaques and NP tau pathology in APP/PS1 mice. Protein p62 is an autophagy receptor specifically degraded by autophagy, which is an important autophagy marker [32]. Accumulation of p62+ puncta was easily observed in vehicle-treated APP/ PS1 mice (Fig. 7a, c), which co-localized with Iba1+ microglia (Fig. 7b). Metformin significantly reduced the accumulation of p62 in both ${ }^{\mathrm{WT}} \mathrm{BE}$-injected and ${ }^{\mathrm{PS} 19} \mathrm{BE}$ - injected APP/PS1 mice (Fig. 7a, c). Besides, p62 immunoreactivity on Iba1+ microglia was also significantly decreased in metformin-treated APP/PS1 mice (Fig. 7b, d, e).

\section{Discussion}

In the current study, we explored the relationship between $A \beta$ accumulation and the propagation of tau pathology and assessed the effects of metformin on both pathologies in APP/PS1 mice. We observed seeded NP tau aggregates in ${ }^{\mathrm{PS} 19} \mathrm{BE}$-injected $\mathrm{APP} / \mathrm{PS} 1$ mice but not in ${ }^{\mathrm{PS} 19} \mathrm{BE}$-injected WT mice or ${ }^{\mathrm{WT}} \mathrm{BE}$-injected APP/PS1 mice. Increased $A \beta$ plaque burden in the cortex and hippocampus and reduced number of microglia around $\mathrm{A} \beta$ plaques were observed in ${ }^{\mathrm{PS} 19} \mathrm{BE}$-injected APP/PS1 mice. Administration of metformin reduced the accumulation of p62 in microglia, increased the number of microglia around $A \beta$ plaques, promoted the internalization of NP tau by microglia, and reduced $A \beta$ pathology as well as NP tau pathology. 


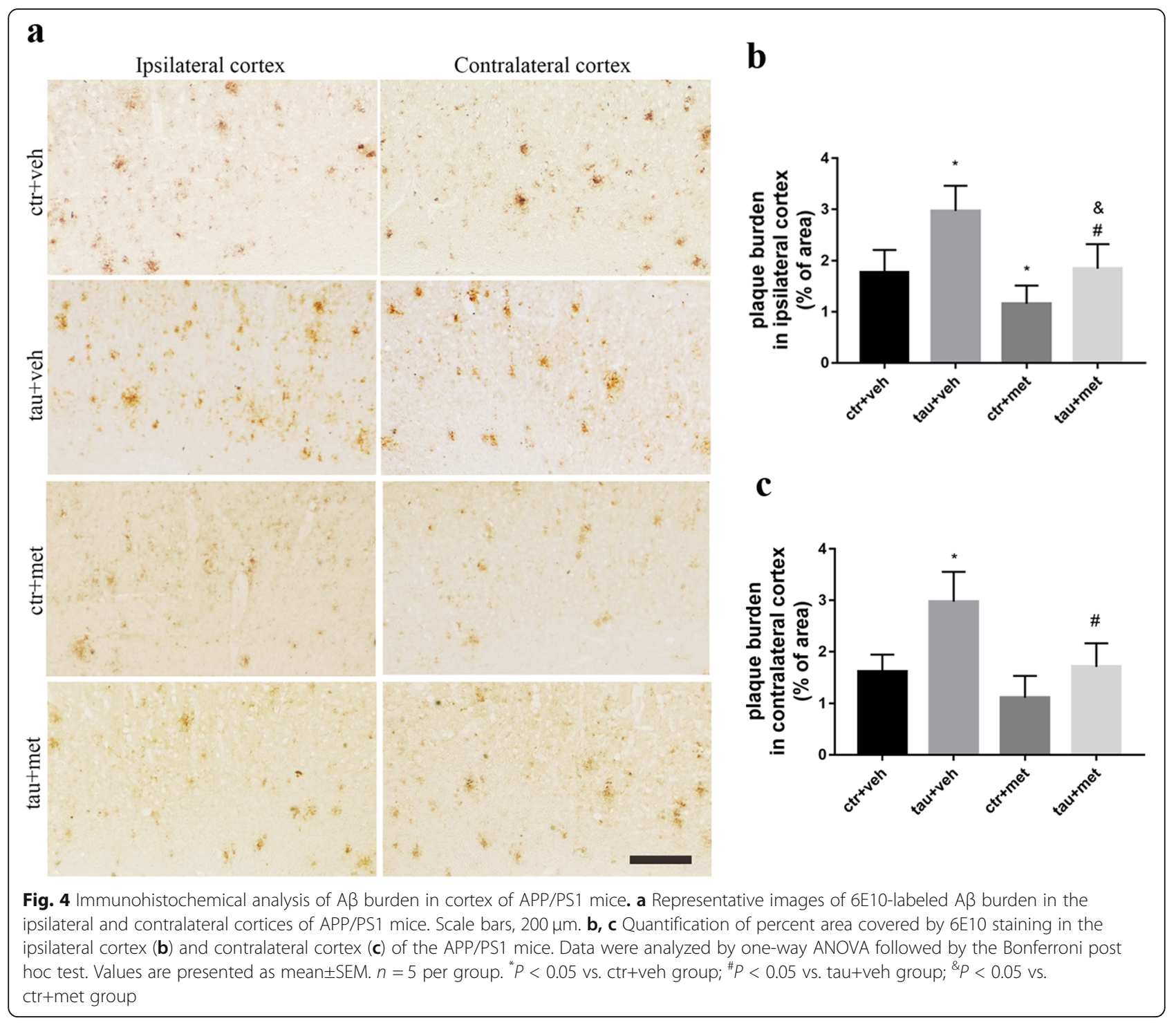

The amyloid cascade theory of AD etiology states that the accumulation of $\mathrm{A} \beta$ plaque precipitates tau pathology [1]. In the amyloid cascade hypothesis, $A \beta$ is described as the upstream of tau aggregation, and tau is considered to be the "bullet" of $A \beta$ [33], responsible for the cytotoxic, dystrophic, and functional effects of $A \beta$ [33-36]. This hypothesis is strongly supported by many studies, which have shown that $A \beta$ pathology could facilitate the progression of tau pathology [37-40]. In the current study, we found that injection of brain extract containing tau aggregates into the brains of APP/PS1 mice induced the formation of NP tau aggregation surrounding $A \beta$ plaques. However, no NP tau pathology was observed in the brains of WT mice after injection of proteopathic tau seeds, suggesting that the presence of $\mathrm{A} \beta$ pathology was necessary for the aggregation of NP tau pathology. A previous study also demonstrated enhancement of tau spreading in the cortex of 5xFAD mice after intracerebral injection of tau seeds [3]. A recent study showed that NP tau pathology was detected 3 months after injection of tau seeds into 8month-old 5xFAD mice and NFT pathology was found 6 months after injection [2]. It is proposed that, at the early seeding stage, $A \beta$ plaques induce the accumulation of endogenous tau within dystrophic neurites surrounding the plaques, leading to the rapid recruitment of proteopathic tau seed into aggregates as NP tau. At this stage, insufficient amounts of proteopathic tau seeds are available to translocate into the neuronal soma dendrites to form NFTs [2]. Consistently, NP tau but not NFT pathology was observed 2 months after injection of ${ }^{\mathrm{PS} 19} \mathrm{BE}$ into the APP/PS1 mice, which is in line with this hypothesis.

In our experiment, proteopathic tau seeds were injected into the dentate gyrus and the overlying 


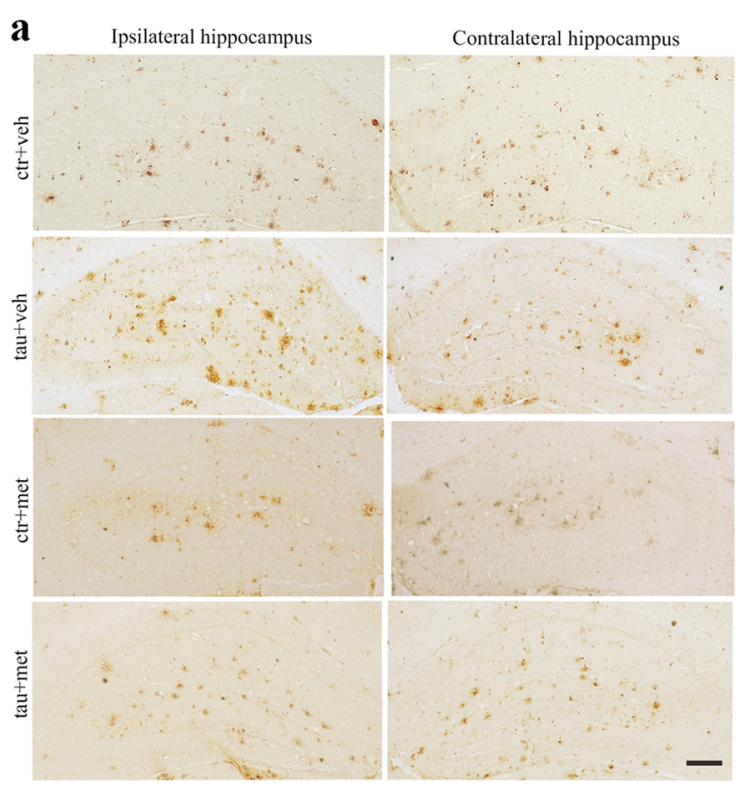

b
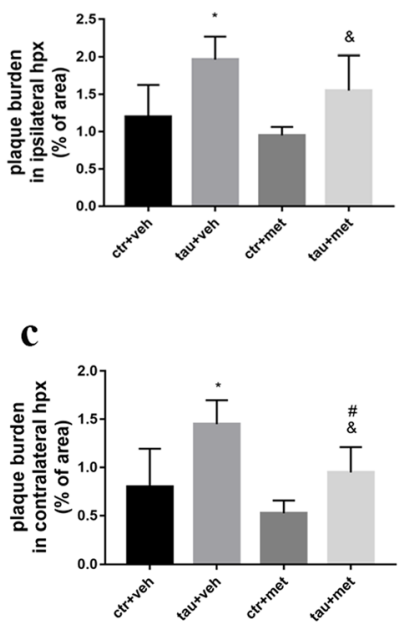

d

f

h
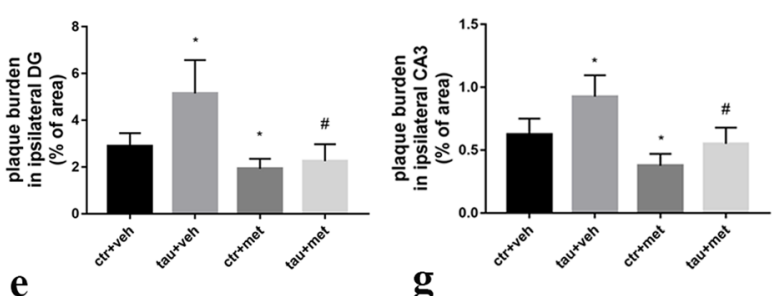

g
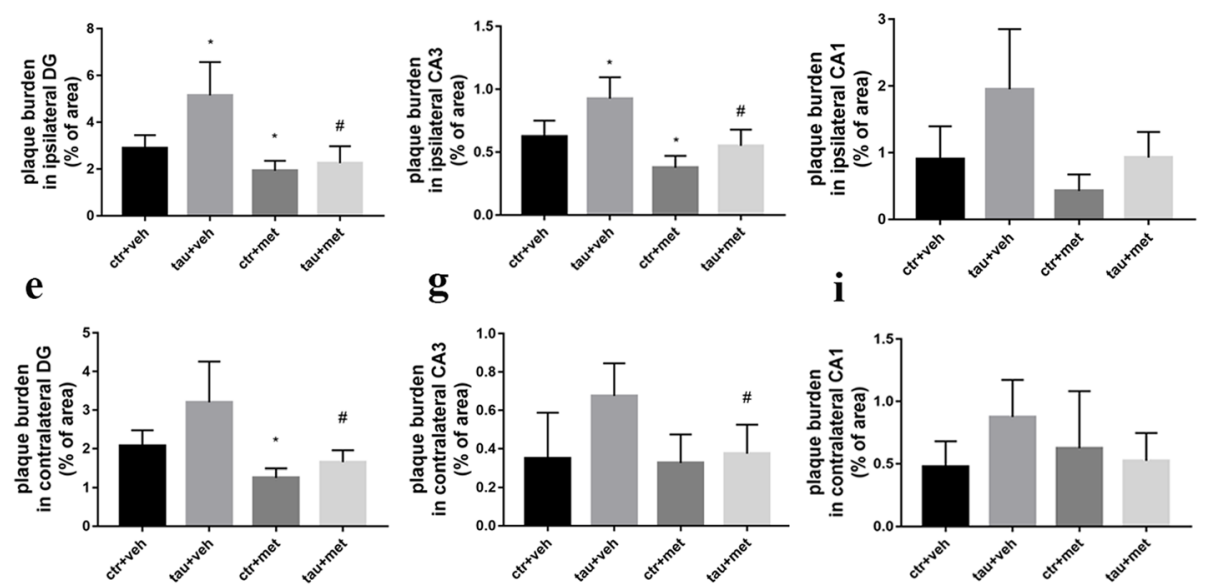

Fig. 5 Immunohistochemical analysis of A burden in hippocampus of APP/PS1 mice. a Representative images of 6E10-labeled A burden in the ipsilateral and contralateral hippocampus of APP/PS1 mice. Scale bars, $200 \mu \mathrm{m}$. b, c Quantification of percent area covered by $6 \mathrm{E} 10$ staining in the ipsilateral hippocampus (hpx) (b) and contralateral hippocampus (c) of the APP/PS1 mice. d-i Quantification of percent area covered by 6 E10 staining in the ipsilateral DG (d), CA3 (f), CA1 (h), contralateral DG (e), CA3 (g), and CA1 (i) of the hippocampi of APP/PS1 mice. Data were analyzed by one-way ANOVA followed by the Bonferroni post hoc test. Values are presented as mean \pm SEM. $n=5$ per group. ${ }^{*} P<0.05$ vs. $c t r+v e h$ group; ${ }^{\#} P<0.05$ vs. tau+veh group; ${ }^{\&} P<0.05$ vs. ctr + met group

cortex of the APP/PS1 mice. Two months after injection, NP tau aggregates were mainly distributed in the dentate gyrus and CA3 region of the ipsilateral hippocampus and overlying cortex of the ${ }^{\mathrm{PS} 19} \mathrm{BE}-$ injected APP/PS1 mice. Small amounts of NP tau aggregates were also detected in the contralateral hippocampus and cortex, indicating that tau pathology had progressed into the contralateral hemisphere. Many studies have demonstrated the trans-synaptic propogation of tau pathology [26, 41, 42]. The hippocampal trisynaptic circuitry (perforant pathway-DG-CA3-CA1) has been well clarified by many studies [43]. In the hippocampal synaptic network, CA3 neurons receive inputs from the DG via mossy fiber (MF) and send axons to the CA1 neurons via Schaffer Collateral Pathway (SC) [43]. Thus, it is possible that NP tau pathology was formed in the DG area and then spread to the CA3 subfield through the synaptic connectivity and later propagated into the contralateral hemisphere.

In the current study, $A \beta$ pathology was not seen in the ${ }^{\mathrm{PS} 19} \mathrm{BE}$-injected WT mice. ${ }^{\mathrm{WT}} \mathrm{BE}$-injected APP/PS1 mice did not show any increase in $A \beta$ pathology. These observations suggest that both APP and tau are necessary for 


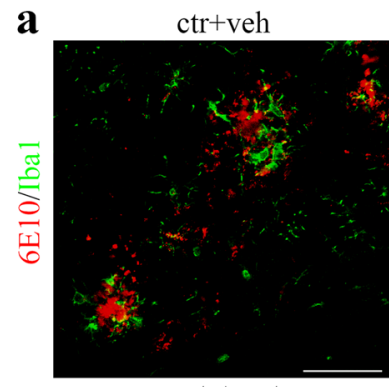

ctr+met

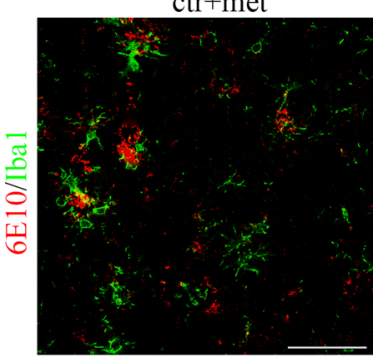

c

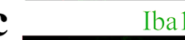

tau+veh

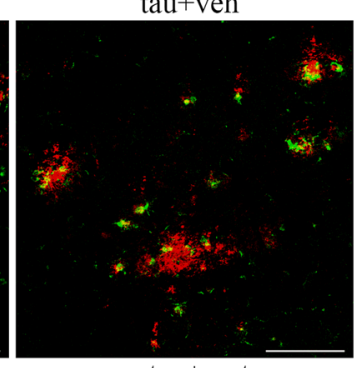

tau+met

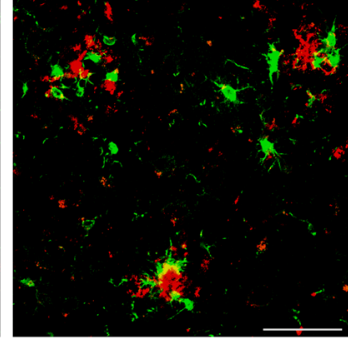

b

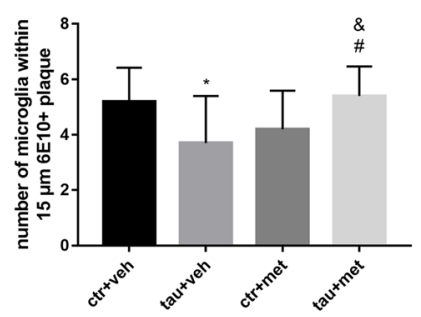

d

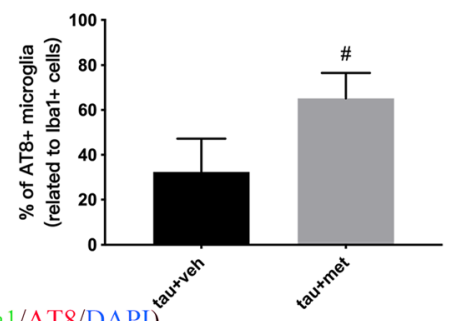

Merged(Ibal/AT8/DAPI)
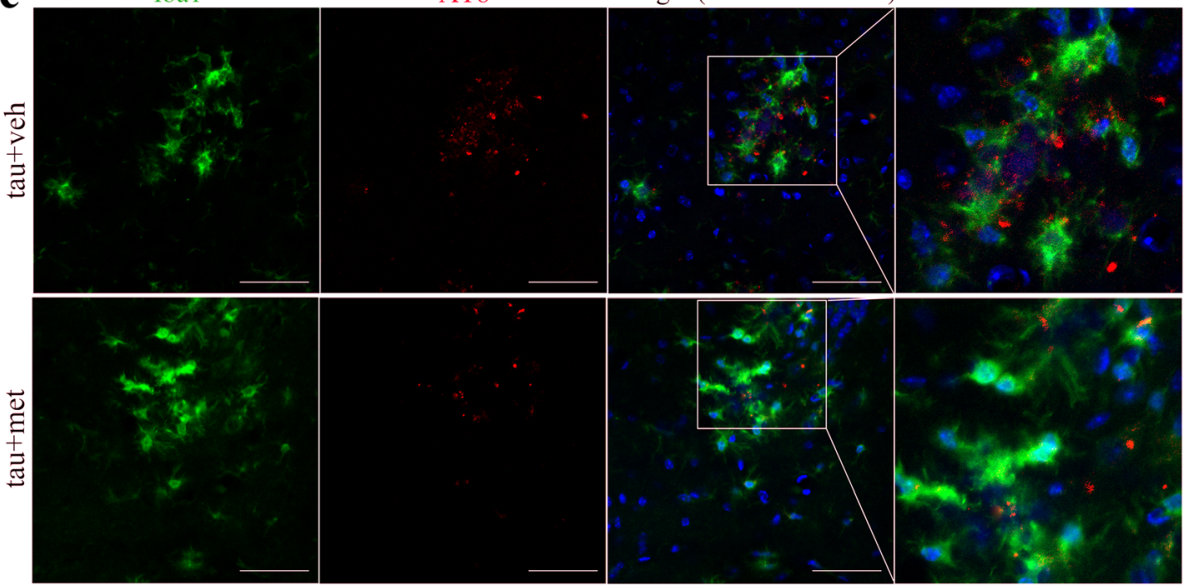

Fig. 6 Immunofluorescence analysis of microglia in relation with A $\beta$ plaques and NP tau pathologies in APP/PS1 mice. a Representative confocal images of Iba1+ microglia (green) surrounding 6E10+ A plaques (red) in the brains of APP/PS1 mice. Scale bars, $100 \mu \mathrm{m}$. b Quantification of the number of microglial cells surrounding plaques in APP/PS1 mice. Data were analyzed by one-way ANOVA followed by the Bonferroni post hoc test. Values are presented as mean \pm SEM. $n=5$ per group. ${ }^{*} P<0.05$ vs. $c t r+v e h$ group; ${ }^{\#} P<0.05$ vs. tau+veh group; ${ }^{\&} P<0.05$ vs. ctr+met group. $\mathbf{c}$ Representative confocal images of Iba1+ microglia (green) and AT8+ NP tau (red) in the brains of APP/PS1 mice. Scale bars, $50 \mu \mathrm{m}$. $\mathbf{d}$ Percentage

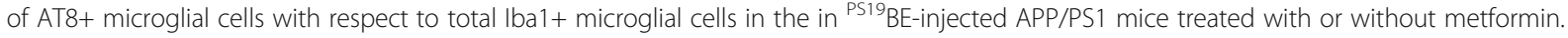
Data were analyzed by Student's $t$ test. Values are presented as mean \pm SEM. $n=5$ per group. ${ }^{\sharp} P<0.05$ vs. tau+veh group

the increase in tau seed-related $A \beta$ aggregation. Interestingly, in ${ }^{\mathrm{PS} 19} \mathrm{BE}$-injected $\mathrm{APP} / \mathrm{PS} 1$ mice, increased $\mathrm{A} \beta$ plaque burden was observed in bilateral cortices and hippocampi, which coincide with the distribution of NP tau aggregates. This observation implies that NP tau pathology can lead to the deterioration of $A \beta$ pathology, challenging the amyloid cascade hypothesis which posits that $A \beta$ acts exclusively upstream of tau pathology and tau pathology is simply a downstream effect of amyloid production in AD. However, the underlying mechanism involved in the accelerated $\mathrm{A} \beta$ deposition in the ${ }^{\mathrm{PS} 19} \mathrm{BE}$ injected APP/PS1 mice remains unclear. Only a few studies have focused on the effects of tau pathology on $A \beta$ accumulation. A previous study showed a fivefold increase of $A \beta$ plaque burden in the brains of double transgenic mice expressing mutated APP and tau in comparison with the mice expressing mutated APP only [44]. Another study demonstrated that tau deletion led to a reduction of A $\beta$ plaque by $50 \%$ in mice expressing mutated APP and PS1 [45]. These data indicate that $A \beta$ and tau may have a positive feedback loop, highlighting synergistic interactions in the development of both lesions. But it is still unknown whether the tau pathology or other related pathological changes accompanied the development of $\mathrm{NP}$, like neuroinflammation, leads to the increase accumulation of $A \beta$ plaque. It has been shown that tau 


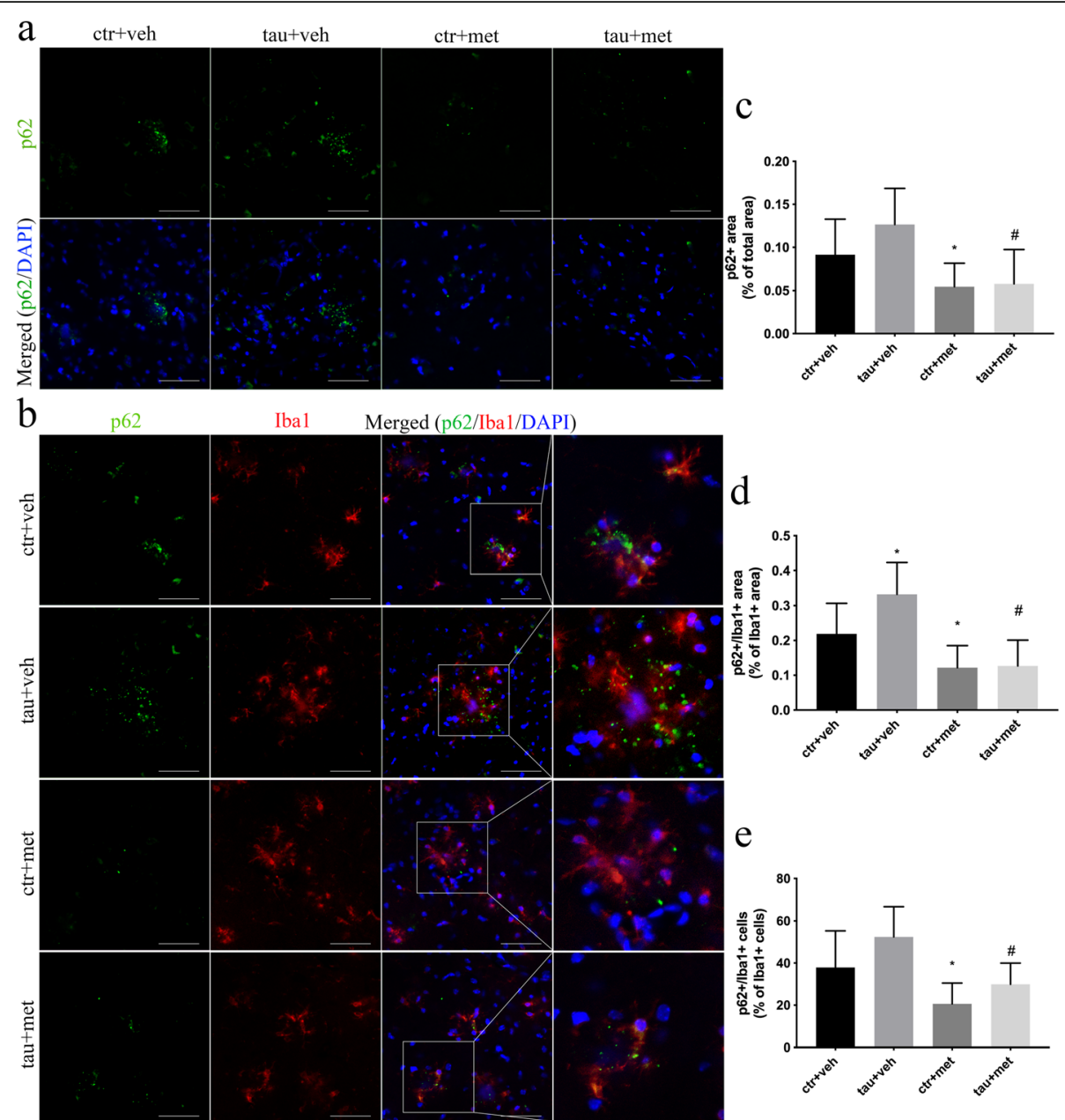

Fig. 7 Metformin reduced accumulation of autophagy-related protein in microglia in APP/PS1 mice. a Representative images of p62+ aggregates (green) in the brains of APP/PS1mice. Scale bars, $50 \mu \mathrm{m}$. b Confocal images of Iba1+ microglia (red) and p62+ aggregates (green) in the brains of APP/PS1 mice. Scale bars, 50 um. c Quantification of percent area covered by p62 staining in APP/PS1 mice. Data were analyzed by one-way ANOVA followed by the Bonferroni post hoc test. Values are presented as mean \pm SEM. $n=5$ per group. $\mathbf{d}$, e Quantitative analysis of p62+ aggregates in microglia, expressed as the percentage of Iba1+ area that is also p62+ (d) and the percentage of the number of Iba $1+$ microglia containing p62+ aggregates (e). Data were analyzed by one-way ANOVA followed by the Bonferroni post hoc test. Values are presented as mean \pm SEM. $n=5$ per group. ${ }^{*} P<0.05$ vs. ctr+veh group; ${ }^{\#} P<0.05$ vs. tau+veh group; ${ }^{\&} P<0.05$ vs. ctr+met group

pathology could induce neuroinflammation, leading to the upregulation of pro-inflammatory cytokines (IL-6, IL-1ß, TNF $\alpha$ ) through NF-kB and MAPK signaling pathways [46]. Therefore, further studies are needed to confirm the extent of neuroinflammation induced by NP tau development and its involvement in the accumulation of $A \beta$ pathology.

Given that the effects of metformin on cognition and AD-related pathologies remain to be determined, we investigated whether metformin could prevent the propagation of $\mathrm{A} \beta$ and tau pathology in ${ }^{\mathrm{PS} 19} \mathrm{BE}$-injected $\mathrm{APP} /$ PS1 mice. We found decreased A $\beta$ burden as well as reduced NP tau pathology in the brains of ${ }^{\mathrm{PS} 19} \mathrm{BE}$-injected APP/PS1 mice treated with metformin, implying that metformin could inhibit the progression of both pathologies. Consistently, it was found that metformin decreased both $A \beta$ deposits and soluble $A \beta$ in APP/PS1 mice [47].

As the immune resident cells of the brain, microglia constantly scan their environment for pathogens [48, 49]. Once activated, microglia can phagocytize cellular debris, produce growth factors, and promote nerve repair. On the other hand, excessive activation of microglia may lead to inflammatory injury to the brain [50]. At early stages of $\mathrm{AD}$, microglia can accumulate around $\mathrm{A} \beta$ plaques and phagocytize $A \beta$ plaques [51], as well as tau [52]. However, if microglia failed to neutralize the seeding activity of tau seeds efficiently, the proteopathic tau seeds would be released from microglia, leading to the acceleration of tau transmission [52]. The diminished phagocytosis of microglia could also lead to the accumulation of both 
pathologies. Here, we observed decreased plaqueassociated microgliosis in ${ }^{\mathrm{PS} 19} \mathrm{BE}$-injected APP/PS1 mice, which might result from the toxicity induced by seeded tau pathology. Administration of metformin increased the number of microglia around $A \beta$ plaques, enhancing plaque-associated microgliosis. We found internalized NP tau inside microglia in the metformin-treated ${ }^{\mathrm{PS} 19} \mathrm{BE}$ injected APP/PS1 mice, which implies that metformin may promote the phagocytosis of tau seeds by microglia, thus reducing the NP tau pathology. These results indicate that metformin may reduce both pathologies by enhancing the phagocytosis of microglia. Consistently, studies have shown that microglia are capable of taking up tau $[52,53]$. It was shown that TREM2-facilitated plaqueassociated microgliosis prevented tau transmission [29]. Thus, it is conceivable that metformin might promote the microglial phagocytosis of $A \beta$ plaque and tau aggregates, limiting the propagation of $A \beta$ and tau pathology. On the other hand, microglia constantly switch between proinflammatory and anti-inflammatory phenotypes under pathological stimulation [54]. Metformin has been shown to decrease the production of pro-inflammatory cytokines in different AD animal models $[47,55]$. Thus, it would be of great interest to further investigate the involvement of neuroinflammatory responses in the reduction of $A \beta$ and tau pathologies with metformin treatment.

Autophagy is a catabolic mechanism of bulk degradation. Pathological proteins and dysfunctional organelles are engulfed to form the autophagosome, which subsequently fuses with lysosomes to form autolysosomes, leading to degradation of autophagic cargoes [56]. Autophagy dysfunction has been observed in $\mathrm{AD}$ brain, contributing to the accumulation of pathological protein aggregates [57]. Metformin is an activator of adenosine monophosphate (AMP)-activated protein kinase (AMPK) $[58,59]$, the activation of which could inhibit the activity of mechanistic target of rapamycin complex 1 (mTORC1) and activate the unc-51 like autophagy activating kinase 1 (ULK-1) [57], hence activating the downstream autophagy pathway. Accumulation of the autophagic substrate p62 in microglia indicates the dysfunction of autophagy degradation [30]. In the current study, we observed accumulation of p62 in microglial cells in the brains of APP/PS1 mice, suggesting the impairment of autophagy. Administration of metformin significantly reduced the accumulation of p62 in microglia, indicating that metformin may ameliorate the microglial autophagy impairment. Consistently, metformin has been shown to increase the microglial capability of phagocytosis in AMPK-dependent manner in rat primary microglia [60]. Pharmacologically inhibiting mTORC1 signaling with rapamycin increased autophagy and ameliorated $A \beta$ and tau pathology [61]. Therefore, it is conceivable that metformin might promote the engulfment of $A \beta$ and tau pathology by enhancing the microglial autophagy capability.

Our study showed that metformin limited the propagation of $\mathrm{A} \beta$ and NP tau pathology in APP/PS1 mice. However, opposing studies have also been reported. It was found that metformin increased the generation of $A \beta$ in various cell models $[62,63]$. These results were observed in cell lines overexpressing APP which is quite different from in vivo condition. In our study, although we could not rule out the effects of metformin on $A \beta$ production in neurons, the reduced $A \beta$ deposition we observed could be resulted from increased microgliosis and the promoted autophagic flux in microglia. Another study also showed increased protein phosphatase 2A (PP2A) level and thus decreased AT8+ staining in P301S mice treated with metformin [25], which is similar to what was found in this study. However, increased insoluble tau species were observed in metformin-treated P301S mouse brain. And the underlying mechanism was unknown. In the current study, we showed decreased NP tau in tau-seeded APP/PS1 mice, suggesting that metformin could interfere with the propagation of tau pathology. These results indicate the potential timewindow for metformin treatment as a therapeutic drug for AD. Metformin might benefit mild cognitive impairment (MCI) or early-stage AD patients. However, further clinical trials are undoubtedly needed to confirm this assumption.

Limitations of the current study include the lack of the behavioral studies and biochemical experiments following injection of proteopathic tau seeds into the APP/PS1 mice. Given the main aim of the present study is to explore the relationship between amyloid and tau pathology in $\mathrm{AD}$ and to determine the effects of metformin on amyloid and tau pathologies, we did not perform behavioral tests or biochemical experiments of these mice. Further studies including behavioral tests are needed to verify the effects of metformin on the cognitive deficits of tau-seeded APP/PS1 mice. Biochemical studies, investigating the activation of AMPK signaling pathway, the involvement of neuroinflammation and autophagic flux in both microglia and neurons, and the phosphorylation of tau at different epitopes and the activation of related tau phosphatase and kinases, will help demonstrate the underlying mechanisms of the beneficial effects of metformin on amyloid and tau pathologies.

\section{Conclusion}

In conclusion, the present study provides novel evidence that proteopathic tau seeds exacerbated $A \beta$ pathology in $\mathrm{APP} / \mathrm{PS} 1$ mice and $\mathrm{A} \beta$ plaques promoted the aggregation of NP tau pathology. Metformin ameliorated microglial autophagy impairment, promoted the phagocytosis of pathological $A \beta$ and tau proteins, and reduced both 
$\mathrm{A} \beta$ deposits and NP tau pathology in tau-seeded APP/ PS1 mice. Our findings highlight a synergistic interaction between $A \beta$ and tau pathology and demonstrate that metformincould limit the propagation of $A \beta$ and NP tau pathology by enhancing microglial autophagy activity.

\section{Abbreviations}

AD: Alzheimer's disease; A 3 : Amyloid- $\beta$; NFTs: Neurofibrillary tangles; APP/ PS1: APPswe/PS1DE9; WT: Wild-type; NP tau: tau aggregates in dystrophic neurites surrounding $A \beta$ plaques; $A D$-tau: $A D$-brain-derived proteopathic tau; NTs: Neuropil threads; T2DM: Type 2 diabetes mellitus; ${ }^{W T}$ BE: Brain extract from WT control mice; ${ }^{\text {PS19}}{ }^{B E}$ : Brain extract containing tau aggregates from PS19 mice; PBS: Phosphate-buffered saline; DAPI: 4',6-Diamidino-2phenylindole; ANOVA: One-way analyses of variance; SEM: Standard error of the mean; DG: Dentate gyrus; MF: Mossy fiber; SC: Schaffer Collateral Pathway; AMPK: Adenosine monophosphate (AMP)-activated protein kinase PP2A: Protein phosphatase 2A; mTORC1: Mechanistic target of rapamycin complex 1; ULK-1: Autophagy activating kinase 1; MCl: Mild cognitive impairment

\section{Acknowledgements}

Not applicable.

\section{Authors' contributions}

$Z \mathrm{~S}, \mathrm{FZ}$, and $\mathrm{CY}$ conceived the overall project and experimental design. ZS and FZ performed the experiments. LZ, ZY, ST, and LK analyzed the data. YY and $T J$ wrote the manuscript. $C Y$ and $Z B$ gave guidance to the experiment and helped to revise the manuscript. All authors read and approved the final manuscript.

\section{Funding}

This study was supported by the National Natural Science Foundation of China (Reference numbers: 81520108010 and 81870826) and the Zhejiang Provincial Natural Science Foundation of China (Reference numbers: LY18H090004 and LY17H090003).

\section{Availability of data and materials}

The datasets used and/or analyzed during the current study are available from the corresponding authors on reasonable request.

\section{Ethics approval and consent to participate}

All procedures were approved by the Institutional Animal Care and Use Committee of Zhejiang University and were performed in accordance with the National Institutes of Health Guide for the Care and Use of Laboratory Animals guidelines for the ethical treatment of animals. Efforts were made to minimize the number of animals used.

\section{Consent for publication}

Not applicable.

\section{Competing interests}

The authors declare that they have no competing interests.

\section{Author details}

'Department of Neurology, The Second Affiliated Hospital, School of Medicine, Zhejiang University, Hangzhou, People's Republic of China. 2Department of Geriatrics, The First Affiliated Hospital, School of Medicine, Zhejiang University, Hangzhou, People's Republic of China.

Received: 21 October 2020 Accepted: 23 December 2020 Published online: 09 February 2021

\section{References}

1. Selkoe DJ, Hardy J. The amyloid hypothesis of Alzheimer's disease at 25 years. EMBO Mol Med. 2016;8(6):595-608.

2. He Z, Guo JL, JD MB, et al. Amyloid-beta plaques enhance Alzheimer's brain tau-seeded pathologies by facilitating neuritic plaque tau aggregation. Nat Med. 2018;24(1):29-38.
3. Vergara C, Houben S, Suain V, et al. Amyloid-beta pathology enhances pathological fibrillary tau seeding induced by Alzheimer PHF in vivo. Acta Neuropathol. 2019;137(3):397-412.

4. Saito T, Mihira N, Matsuba $Y$, et al. Humanization of the entire murine Mapt gene provides a murine model of pathological human tau propagation. J Biol Chem. 2019:294(34):12754-65.

5. Herrup K. The case for rejecting the amyloid cascade hypothesis. Nat Neurosci. 2015;18(6):794-9.

6. Giacobini E, Gold G. Alzheimer disease therapy--moving from amyloid-beta to tau. Nat Rev Neurol. 2013;9(12):677-86.

7. Braak H, Braak E. Staging of Alzheimer's disease-related neurofibrillary changes. Neurobiol Aging. 1995;16(3):271-8. discussion 278-284.

8. Hasegawa M, Nonaka T, Masuda-Suzukake M. Prion-like mechanisms and potential therapeutic targets in neurodegenerative disorders. Pharmacol Ther. 2017:172:22-33.

9. Soto C. In vivo spreading of tau pathology. Neuron. 2012;73(4):621-3.

10. El Khoury NB, Gratuze M, Papon MA, Bretteville A, Planel E. Insulin dysfunction and Tau pathology. Front Cell Neurosci. 2014;8:22.

11. Cohen AD, Klunk WE. Early detection of Alzheimer's disease using PiB and FDG PET. Neurobiol Dis. 2014;72:117-22.

12. Talbot $\mathrm{K}$, Wang $\mathrm{HY}$, Kazi $\mathrm{H}$, et al. Demonstrated brain insulin resistance in Alzheimer's disease patients is associated with IGF-1 resistance, IRS-1 dysregulation, and cognitive decline. J Clin Invest. 2012;122(4):1316-38.

13. Rotermund C, Machetanz G, Fitzgerald JC. The therapeutic potential of metformin in neurodegenerative diseases. Front Endocrinol (Lausanne). 2018;9:400.

14. Hsu CC, Wahlqvist ML, Lee MS, Tsai HN. Incidence of dementia is increased in type 2 diabetes and reduced by the use of sulfonylureas and metformin. J Alzheimers Dis. 2011;24(3):485-93.

15. Cheng C, Lin CH, Tsai YW, Tsai CJ, Chou PH, Lan TH. Type 2 diabetes and antidiabetic medications in relation to dementia diagnosis. J Gerontol A Biol Sci Med Sci. 2014;69(10):1299-305.

16. Orkaby AR, Cho K, Cormack J, Gagnon DR, Driver JA. Metformin vs sulfonylurea use and risk of dementia in US veterans aged $>/=65$ years with diabetes. Neurology. 2017;89(18):1877-85.

17. Luchsinger JA, Perez $\mathrm{T}$, Chang $\mathrm{H}$, et al. Metformin in amnestic mild cognitive impairment: results of a pilot randomized placebo controlled clinical trial. J Alzheimers Dis. 2016:51(2):501-14.

18. Imfeld P, Bodmer M, Jick SS, Meier CR. Metformin, other antidiabetic drugs, and risk of Alzheimer's disease: a population-based case-control study. J Am Geriatr Soc. 2012;60(5):916-21.

19. Wang C-P, Lorenzo C, Habib SL, Jo B, Espinoza SE. Differential effects of metformin on age related comorbidities in older men with type 2 diabetes. J Diab Complications. 2017;31(4):679-86.

20. Oliveira WH, Nunes AK, Franca ME, et al. Effects of metformin on inflammation and short-term memory in streptozotocin-induced diabetic mice. Brain Res. 2016;1644:149-60.

21. Li J, Deng J, Sheng W, Zuo Z. Metformin attenuates Alzheimer's disease-like neuropathology in obese, leptin-resistant mice. Pharmacol Biochem Behav. 2012;101(4):564-74.

22. Pintana H, Apaijai N, Pratchayasakul W, Chattipakorn N, Chattipakorn SC Effects of metformin on learning and memory behaviors and brain mitochondrial functions in high fat diet induced insulin resistant rats. Life Sci. 2012;91(11-12):409-14.

23. Chen JL, Luo C, Pu D, et al. Metformin attenuates diabetes-induced tau hyperphosphorylation in vitro and in vivo by enhancing autophagic clearance. Exp Neurol. 2019:311:44-56.

24. Kickstein E, Krauss $S$, Thornhill P, et al. Biguanide metformin acts on tau phosphorylation via mTOR/protein phosphatase 2A (PP2A) signaling. Proc Natl Acad Sci U S A. 2010;107(50):21830-5.

25. Barini $E$, Antico $O$, Zhao $Y$, et al. Metformin promotes tau aggregation and exacerbates abnormal behavior in a mouse model of tauopathy. Mol Neurodegener. 2016;11:16.

26. Ahmed Z, Cooper J, Murray TK, et al. A novel in vivo model of tau propagation with rapid and progressive neurofibrillary tangle pathology: the pattern of spread is determined by connectivity, not proximity. Acta Neuropathol. 2014;127(5):667-83.

27. Chen $Y$, Zhao $Y$, Dai $C L$, et al. Intranasal insulin restores insulin signaling, increases synaptic proteins, and reduces Abeta level and microglia activation in the brains of 3xTg-AD mice. Exp Neurol. 2014; 261:610-9. 
28. Mao YF, Guo Z, Zheng T, et al. Intranasal insulin alleviates cognitive deficits and amyloid pathology in young adult APPswe/PS1dE9 mice. Aging Cell. 2016;15(5):893-902.

29. CEG L, Gratuze M, Narasimhan S, et al. TREM2 function impedes tau seeding in neuritic plaques. Nat Neurosci. 2019;22(8):1217-22.

30. Pomilio C, Gorojod RM, Riudavets M, et al. Microglial autophagy is impaired by prolonged exposure to beta-amyloid peptides: evidence from experimental models and Alzheimer's disease patients. Geroscience. 2020; 42(2):613-32.

31. Long Z, Chen J, Zhao Y, et al. Dynamic changes of autophagic flux induced by Abeta in the brain of postmortem Alzheimer's disease patients, animal models and cell models. Aging. 2020;12(11):10912-30.

32. Spilman P, Podlutskaya N, Hart MJ, et al. Inhibition of mTOR by rapamycin abolishes cognitive deficits and reduces amyloid-beta levels in a mouse model of Alzheimer's disease. Plos One. 2010:5(4):e9979.

33. Bloom GS. Amyloid-beta and tau: the trigger and bullet in Alzheimer disease pathogenesis. JAMA Neurol. 2014;71(4):505-8.

34. Roberson ED, Scearce-Levie K, Palop JJ, et al. Reducing endogenous tau ameliorates amyloid beta-induced deficits in an Alzheimer's disease mouse model. Science. 2007;316(5825):750-4.

35. Chabrier MA, Cheng D, Castello NA, Green KN, FM LF. Synergistic effects of amyloid-beta and wild-type human tau on dendritic spine loss in a floxed double transgenic model of Alzheimer's disease. Neurobiol Dis. 2014;64:107-17.

36. Jin M, Shepardson N, Yang T, Chen G, Walsh D, Selkoe DJ. Soluble amyloid beta-protein dimers isolated from Alzheimer cortex directly induce Tau hyperphosphorylation and neuritic degeneration. Proc Natl Acad Sci U S A. 2011;108(14):5819-24.

37. Hurtado DE, Molina-Porcel L, Iba M, et al. A \{beta\} accelerates the spatiotemporal progression of tau pathology and augments tau amyloidosis in an Alzheimer mouse model. Am J Pathol. 2010;177(4):1977-88.

38. Pooler AM, Polydoro M, Maury EA, et al. Amyloid accelerates tau propagation and toxicity in a model of early Alzheimer's disease. Acta Neuropathol Commun. 2015;3:14

39. Bennett RE, SL DV, Dujardin S, et al. Enhanced tau aggregation in the presence of amyloid beta. Am J Pathol. 2017;187(7):1601-12.

40. Saul A, Sprenger F, Bayer TA, Wirths O. Accelerated tau pathology with synaptic and neuronal loss in a novel triple transgenic mouse model of Alzheimer's disease. Neurobiol Aging. 2013;34(11):2564-73.

41. de Calignon A, Polydoro M, Suarez-Calvet M, et al. Propagation of tau pathology in a model of early Alzheimer's disease. Neuron. 2012;73(4): 685-97.

42. Liu L, Drouet $\mathrm{V}, \mathrm{Wu}$ JW, et al. Trans-synaptic spread of tau pathology in vivo. Plos One. 2012;7(2):e31302.

43. Lee I, Kesner RP. Encoding versus retrieval of spatial memory: double dissociation between the dentate gyrus and the perforant path inputs into CA3 in the dorsal hippocampus. Hippocampus. 2004;14(1):66-76.

44. Ribe EM, Perez M, Puig B, et al. Accelerated amyloid deposition, neurofibrillary degeneration and neuronal loss in double mutant APP/tau transgenic mice. Neurobiol Dis. 2005;20(3):814-22.

45. Leroy $K$, Ando $K$, Laporte $V$, et al. Lack of tau proteins rescues neuronal cel death and decreases amyloidogenic processing of APP in APP/PS1 mice. Am J Pathol. 2012;181(6):1928-40.

46. Kovac A, Zilka N, Kazmerova Z, Cente M, Zilkova M, Novak M. Misfolded truncated protein tau induces innate immune response via MAPK pathway. J Immunol. 2011;187(5):2732-9.

47. Ou Z, Kong X, Sun $X$, et al. Metformin treatment prevents amyloid plaque deposition and memory impairment in APP/PS1 mice. Brain Behav Immun. 2018;69:351-63.

48. Nimmerjahn A, Kirchhoff F, Helmchen F. Resting microglial cells are highly dynamic surveillants of brain parenchyma in vivo. Science. 2005;308(5726): 1314-8.

49. Tremblay ME. The role of microglia at synapses in the healthy CNS: novel insights from recent imaging studies. Neuron Glia Biol. 2011;7(1):67-76.

50. Hanisch UK, Kettenmann H. Microglia: active sensor and versatile effector cells in the normal and pathologic brain. Nat Neurosci. 2007;10(11):1387-94.

51. Condello C, Yuan P, Schain A, Grutzendler J. Microglia constitute a barrier that prevents neurotoxic protofibrillar Abeta42 hotspots around plaques. Nat Commun. 2015;6:6176.

52. Hopp SC, Lin Y, Oakley D, et al. The role of microglia in processing and spreading of bioactive tau seeds in Alzheimer's disease. $J$ Neuroinflammation. 2018;15(1):269.
53. Bolos M, Llorens-Martin M, Jurado-Arjona J, Hernandez F, Rabano A, Avila J. Direct evidence of internalization of tau by microglia in vitro and in vivo. J Alzheimers Dis. 2016;50(1):77-87.

54. Tang $Y$, Le $W$. Differential roles of $M 1$ and $M 2$ microglia in neurodegenerative diseases. Mol Neurobiol. 2016;53(2):1181-94.

55. Saffari PM, Alijanpour S, Takzaree N, et al. Metformin loaded phosphatidylserine nanoliposomes improve memory deficit and reduce neuroinflammation in streptozotocin-induced Alzheimer's disease model. Life Sci. 2020;255:117861.

56. Zhao YG, Zhang $H$. Autophagosome maturation: an epic journey from the ER to lysosomes. J Cell Biol. 2019;218(3):757-70.

57. Plaza-Zabala A, Sierra-Torre V, Sierra A. Autophagy and microglia: novel partners in neurodegeneration and aging. Int J Mol Sci. 2017;18(3):598.

58. Hettich MM, Matthes F, Ryan DP, et al. The anti-diabetic drug metformin reduces BACE1 protein level by interfering with the MID1 complex. Plos One. 2014;9(7):e102420.

59. Salminen A, Kaarniranta K, Haapasalo A, Soininen H, Hiltunen M. AMPactivated protein kinase: a potential player in Alzheimer's disease. J Neurochem. 2011;118(4):460-74.

60. Labuzek K, Liber S, Gabryel B, Adamczyk J, Okopien B. Metformin increases phagocytosis and acidifies lysosomal/endosomal compartments in AMPKdependent manner in rat primary microglia. Naunyn Schmiedebergs Arch Pharmacol. 2010;381(2):171-86

61. Caccamo A, Majumder S, Richardson A, Strong R, Oddo S. Molecular interplay between mammalian target of rapamycin (mTOR), amyloid-beta, and Tau: effects on cognitive impairments. J Biol Chem. 2010;285(17):13107-20.

62. Chen Y, Zhou K, Wang R, et al. Antidiabetic drug metformin (GlucophageR) increases biogenesis of Alzheimer's amyloid peptides via up-regulating BACE1 transcription. Proc Natl Acad Sci U S A. 2009;106(10):3907-12.

63. Son SM, Shin HJ, Byun J, et al. Metformin facilitates amyloid-beta generation by beta- and gamma-secretases via autophagy activation. J Alzheimers Dis. 2016;51(4):1197-208

\section{Publisher's Note}

Springer Nature remains neutral with regard to jurisdictional claims in published maps and institutional affiliations.

Ready to submit your research? Choose BMC and benefit from:

- fast, convenient online submission

- thorough peer review by experienced researchers in your field

- rapid publication on acceptance

- support for research data, including large and complex data types

- gold Open Access which fosters wider collaboration and increased citations

- maximum visibility for your research: over $100 \mathrm{M}$ website views per year

At BMC, research is always in progress.

Learn more biomedcentral.com/submissions 